\title{
CT Perfusion for Detection of Delayed Cerebral Ischemia in Aneurysmal Subarachnoid Hemorrhage: A Systematic Review and Meta-Analysis
}

D.I.A. Mir, A. Gupta, A. Dunning, L. Puchi, C.L. Robinson, H.-A.B. Epstein, and P.C. Sanelli

\begin{abstract}
BACKGROUND AND PURPOSE: Delayed cerebral ischemia is a significant cause of morbidity and mortality after aneurysmal SAH, leading to poor outcomes. The purpose of this study was to evaluate the usefulness of CTP in determining delayed cerebral ischemia in patients with aneurysmal SAH.
\end{abstract}

MATERIALS AND METHODS: We conducted a systematic review evaluating studies that assessed CTP in patients with aneurysmal SAH for determining delayed cerebral ischemia. Studies using any of the following definitions of delayed cerebral ischemia were included in the systematic review: 1) new onset of clinical deterioration, 2) cerebral infarction identified on follow-up CT or MR imaging, and 3) functional disability. A random-effects meta-analysis was performed assessing the strength of association between a positive CTP result and delayed cerebral ischemia.

RESULTS: The systematic review identified 218 studies that met our screening criteria, of which 6 cohort studies met the inclusion criteria. These studies encompassed a total of 345 patients, with 155 (45\%) of 345 patients classified as having delayed cerebral ischemia and 190 (55\%) of 345 patients as not having delayed cerebral ischemia. Admission disease severity was comparable across all groups. Four cohort studies reported CTP test characteristics amenable to the meta-analysis. The weighted averages and ranges of the pooled sensitivity and specificity of CTP in the determination of delayed cerebral ischemia were $0.84(0.7-0.95)$ and $0.77(0.66-0.82)$, respectively. The pooled odds ratio of $23.14(95 \% \mathrm{Cl}, 5.87-91.19)$ indicates that patients with aneurysmal SAH with positive CTP test results were approximately 23 times more likely to experience delayed cerebral ischemia compared with patients with negative CTP test results.

CONCLUSIONS: Perfusion deficits on CTP are a significant finding in determining delayed cerebral ischemia in aneurysmal SAH. This may be helpful in identifying patients with delayed cerebral ischemia before development of infarction and neurologic deficits.

ABBREVIATIONS: DCI = delayed cerebral ischemia; QUADAS = Quality Assessment of Diagnostic Accuracy Studies

A neurysmal SAH is a devastating condition that occurs in up to 30,000 people in the United States annually and carries a $51 \%$ case mortality. ${ }^{1,2}$ Delayed cerebral ischemia (DCI) is consid-

Received July 30, 2013; accepted after revision September 19.

From the Departments of Radiology (D.I.A.M., A.G., L.P., C.L.R., P.C.S.), and Public Health (A.D., P.C.S.), and the Samuel J. Wood Library and C.V. Starr Biomedical Information Center (H.-A.B.E.), Weill Cornell Medical College, New York, NY.

This publication was supported by Grant Number 5K23NS058387 from the National Institute of Neurological Disorders and Stroke (NINDS), a component of the National Institutes of Health $(\mathrm{NIH})$. Its contents are solely the responsibility of the authors and do not necessarily represent the official view of NINDS or NIH.

Please address correspondence to Pina C. Sanelli, MD, MPH, Department of Radiology, Weill Cornell Medical College/New York Presbyterian Hospital, 525 E. 68th St, Starr 8A, New York, NY 10065; e-mail: pcs9001@med.cornell.edu

- Indicates open access to non-subscribers at www.ajnr.org

三 Indicates article with supplemental on-line appendix.

EBM Evidence-Based Medicine Level 2.

http://dx.doi.org/10.3174/ajnr.A3787 ered the most significant cause of morbidity and mortality in patients who survive the initial hemorrhage, with poor outcomes occurring in up to $30 \%$ despite aggressive therapy. ${ }^{3,4}$ The definition of DCI is variable and has been described as a new onset of clinical deterioration, not explained by other causes. ${ }^{5}$ However, recently it has been recommended to define DCI on the basis of its primary outcome measures, such as cerebral infarction and functional disability. ${ }^{6}$ Therefore, DCI is a challenging diagnosis to make prospectively, before its poor outcomes, particularly in comatose or sedated patients, thus limiting initiation of pre-emptive therapy. ${ }^{5}$ Despite these difficulties, DCI is used to complement older clinicoradiographic terminology such as angiographic vasospasm and symptomatic vasospasm because DCI has been shown to have the strongest associations with poor outcomes, including cognitive impairment and reduced quality of life after aneurysmal SAH. ${ }^{6}$ Furthermore, it has become clear that the pathogenesis of DCI is not fully attributable to large-vessel vasospasm alone and 
may, in fact, be the result of several independent processes acting in concert. ${ }^{7}$ The common denominator, however, seems to be hemodynamic alterations in cerebral perfusion leading to ischemia and/or infarction.

Cerebral perfusion can be assessed by use of CT, MR, PET, and SPECT imaging. Specifically, CTP is a technique that allows for rapid, noninvasive assessment of $\mathrm{CBF}, \mathrm{MTT}$, and $\mathrm{CBV}$. CTP has had an increasing role in the evaluation of patients with aneurysmal SAH, as it can be performed in conjunction with traditional noncontrast CT and CTA, requiring little extra examination time. ${ }^{8}$ Many initial studies have evaluated the diagnostic accuracy of CTP for vasospasm compared with DSA- and CTAdefined vasospasm. However, CTP remains a relatively new technique for the evaluation of DCI, and it remains unclear how well CTP can detect DCI. ${ }^{9}$ Given the limitations of relying on the conclusions of individual studies in the literature, particularly when relatively small cohorts are used and findings are not entirely conclusive, we aimed to perform a systematic review and meta-analysis to evaluate CTP in the detection of DCI in patients with aneurysmal SAH.

\section{MATERIALS AND METHODS}

We have implemented the methods described in the Preferred Reporting Items for Systematic Reviews and Meta-Analyses statement. ${ }^{10}$

\section{Eligibility Criteria}

To be more inclusive in identifying studies in the literature that evaluated CTP for DCI in patients with aneurysmal SAH, we included any of the following definitions of DCI in our inclusion criteria: 1) clinical deterioration not explained by other causes and sufficiently judged to be the result of DCI, 2) cerebral infarction identified on follow-up CT or MR imaging, and 3) functional disability related to DCI. If any one of these criteria was positive, DCI was considered to have occurred. We limited our selection to include manuscripts published in English only. In cases of duplicated cohorts, we included the study with the largest number of patients.

\section{Information Sources and Search}

An experienced medical librarian performed a systematic search to identify studies in accordance with the eligibility criteria. Potential articles were found by a search of the electronic data bases of Ovid MEDLINE, EMBASE, and Web of Knowledge. Additional records were identified by use of the Related Articles feature in PubMed and the Cited Reference Search in the ISI Web of Science. All studies included in these data bases through March 2013 were searched. Further details of the search strategy are described in the On-line Appendix.

\section{Study Selection and Data Collection Process}

After removal of duplicate articles, search results were preliminarily screened via title and abstract information by a single reader. Shortlisted manuscripts were independently reviewed in full by 2 additional readers to determine conformity to the eligibility criteria. Disagreements were resolved by consensus.

With use of a standardized data collection template, study characteristics including baseline patient demographics, CTP test characteristics, and detailed DCI outcome data were collected by 2 independent readers, with disagreements resolved by a third reader. All eligible studies were included in the qualitative systematic review. Of these, studies reporting test characteristics or data from which they could be tabulated were included in the meta-analysis.

\section{Validity Assessment}

To assess the validity of each study, we implemented the Quality Assessment of Diagnostic Accuracy Studies (QUADAS) tool. ${ }^{11}$ This tool assesses bias in each study according to 14 criteria, with possible answers as "Yes," "No," or "Unknown." Two reviewers scored each study with disagreements resolved by consensus. The full QUADAS tool is available in the On-line Appendix.

\section{Statistical Analysis}

A meta-analysis was performed on those studies in which test characteristics could be extracted and/or tabulated. Pooled sensitivity, specificity, and odds ratios with corresponding 95\% CIs were calculated. The pooled sensitivity and specificity were calculated by use of weighted averages derived from the sample size of each study. The pooled odds ratio was calculated by use of a random-effects model. We chose this statistical method rather than the less conservative fixed-effects model because of the wide variation in these studies, including the definitions of DCI, different CTP protocols, and postprocessing software, as well as differences in what constituted an abnormal CTP result. Furthermore, study heterogeneity was assessed by calculating the $I^{2}$ statistic. All analyses were conducted by using StataVersion 12 software (StataCorp, College Station, Texas).

\section{RESULTS}

\section{Study Selection}

After removal of duplicated citations, search results yielded 218 manuscripts. Of these, 25 articles were selected for full review on the basis of screening the titles and abstracts, with 14 meeting the eligibility criteria. Seventy-one percent (10/14) of these studies were suspected to contain at least partially overlapping cohorts; therefore, their authors were contacted for further clarification. $^{12-21}$ One group replied to our request (authors of ${ }^{14,16,20,21}$ ) and indicated that duplicate cohorts were used; in this case, the study with the largest number of included patients was included in our review. ${ }^{14}$ No reply was received from the other groups; therefore, only those with the largest cohorts were included. ${ }^{12,13}$ One study was also excluded for not providing the number of patients in the DCI and non-DCI groups. ${ }^{22}$ Finally, 6 studies were included in the systematic review. ${ }^{12-14,23-25}$ The study selection is summarized in Fig 1.

\section{Qualitative Assessment and Study Characteristics}

The 6 studies that met our final eligibility criteria were all prospective, with 3 completed in the United States, ${ }^{14,23,24} 2$ in the Netherlands, ${ }^{12,13}$ and 1 study in Germany. ${ }^{25}$ Together, these studies encompassed 345 patients, of which 155 (45\%) were classified as having DCI and 190 (55\%) as not having DCI. Although admission disease severity, demographics, and median day of DCI onset were comparable across groups, large 

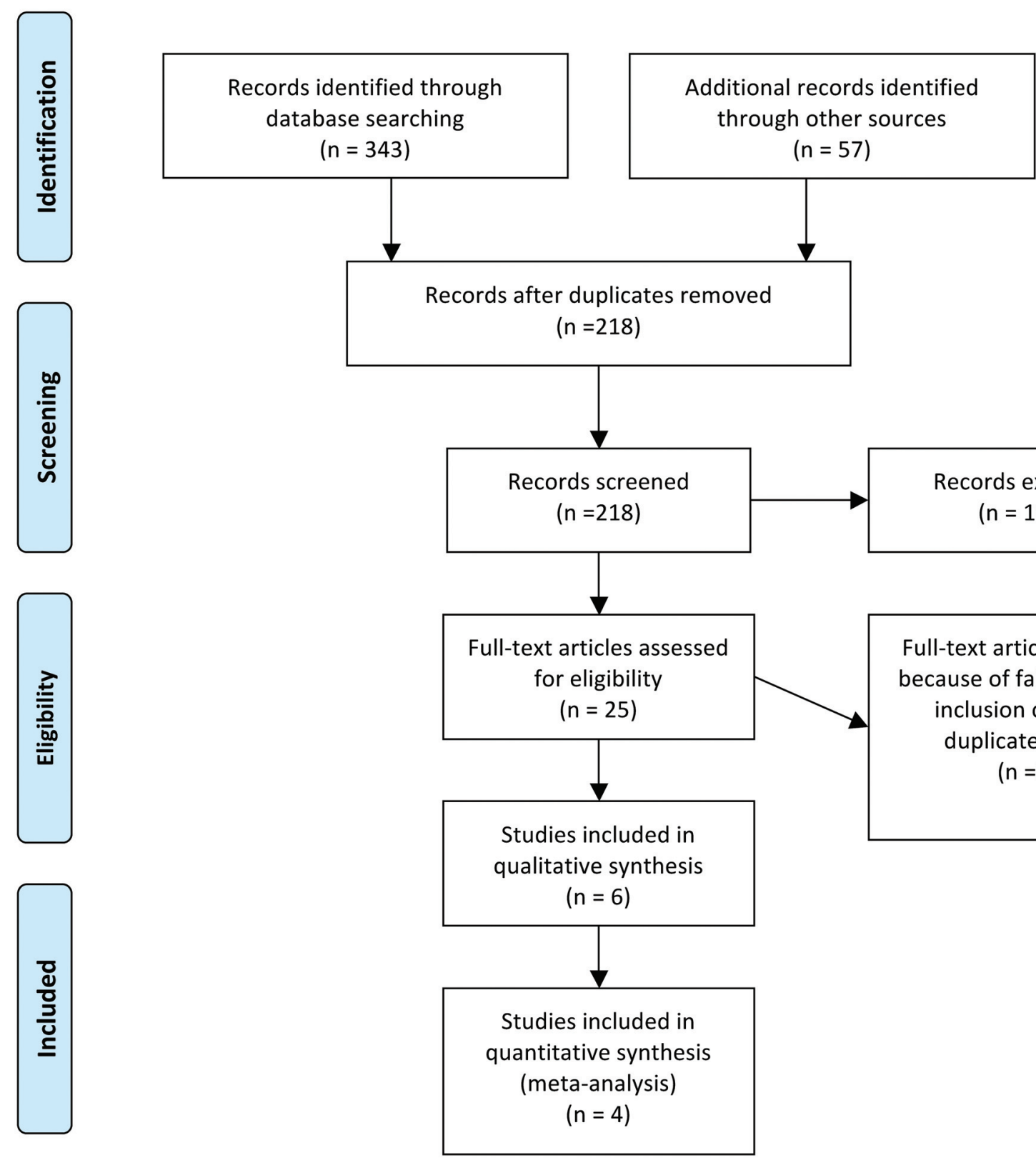

Records excluded

$(n=193)$

FIG 1. Study selection flow diagram. Figure adapted from the Preferred Reporting Items for Systematic Reviews and Meta-Analyses Group Statement. ${ }^{10}$

differences existed in the cohort sizes with a range from 10-97 patients (On-line Table 1). Studies tended to implement CTP imaging either at the onset of symptomatic vasospasm or before; however, for 2 studies this information was not reported. ${ }^{12,24}$ Studies had variable CTP methodology and postprocessing algorithms, variation in the definition of a positive CTP test result, and variability in the definition of DCI used as an outcome measure (On-line Table 2).

\section{Validity Assessment}

The percentage of studies scoring "Yes," "No," or "Unknown" for each of the 14 items on the QUADAS tool is displayed in Fig 2. All of the studies scored "Yes" on items 1 and 8 of the QUADAS tool, indicating an absence of spectrum bias (presence of a cohort representative of patients who would receive the index test in practice) and the presence of a clear description of the definition of
DCI. In addition, $80 \%$ of studies scored "Yes" on items 2, 3, 5-7, 9, and 12 on the QUADAS tool, indicating that a clear description of selection criteria was provided; appropriate reference standards were chosen; and that there was an absence of primary selection bias (whole or part of the study population received verification by the reference standard), differential verification bias (study population received verification by the same reference standard), and incorporation bias (the reference standard was independent of the index test) among most studies. Last, most studies scored "No" or "Unknown" on items 4, 11,13 , and 14, indicating the presence of disease progression bias (unsure if the time between implementation of the reference standard and the index test is short enough to ensure that the target condition did not change between the 2 tests), test classification bias (the reference standard results were interpreted without 


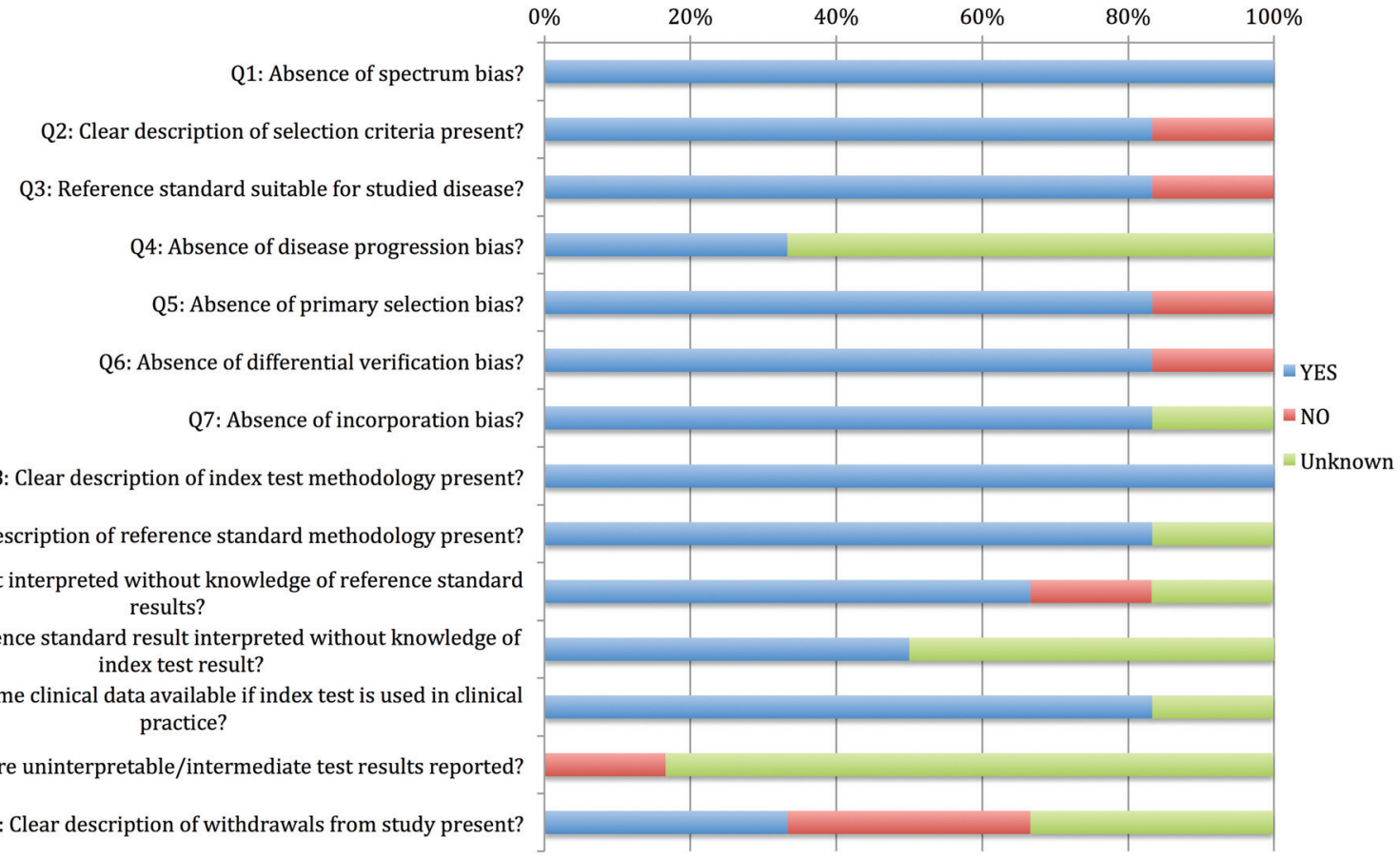

FIG 2. Percentage of studies included in the systematic review scoring "Yes," "No," or "Unknown" on QUADAS tool criteria. Questions truncated in figure according to information provided from previously published work."

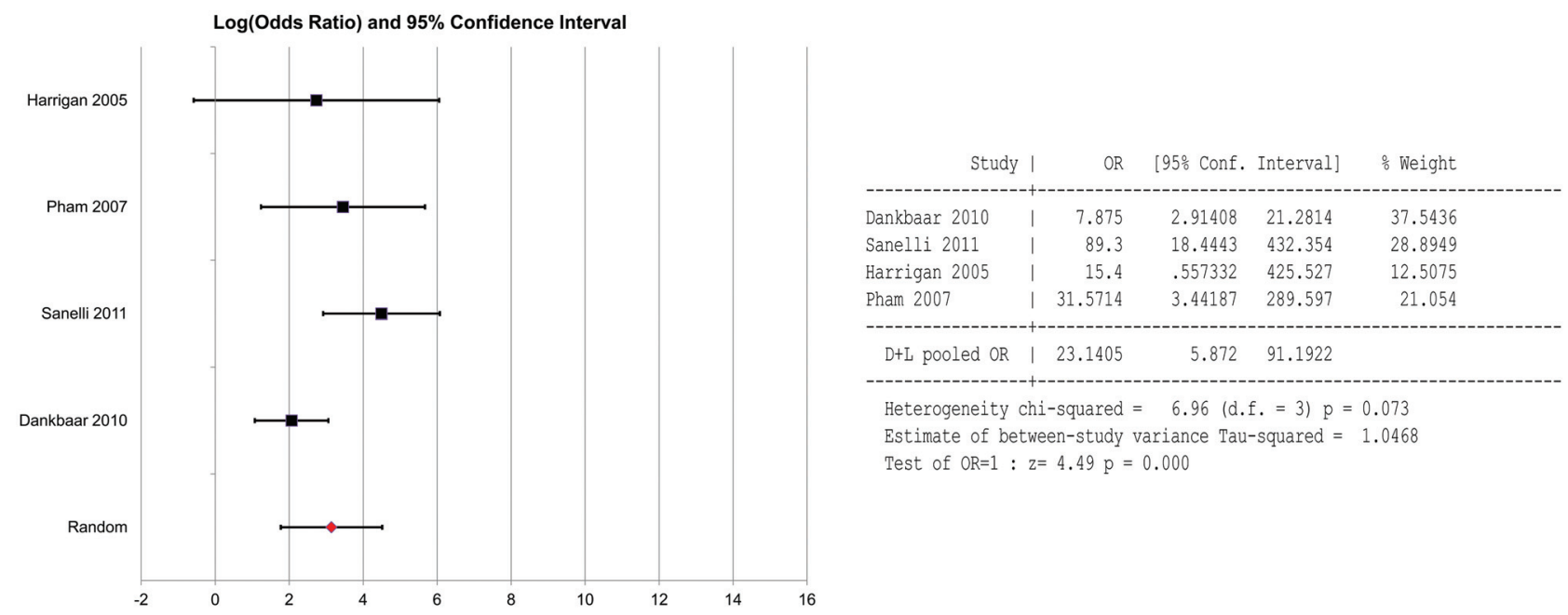

FIG 3. Forest plot of data used to tabulate the pooled odds ratios for studies included in the meta-analysis.

knowledge of the results of the index test and vice versa), and a lack of explanation for patient withdrawals.

\section{Statistical Analysis}

In $4(67 \%)$ of the 6 studies, CTP test characteristics and DCI were reported in a way that could be tabulated for a meta-analysis. ${ }^{13,14,23,25}$ This number included studies attempting to determine optimal CTP characteristics and thresholds to detect DCI; in these cases, we used the best single test characteristic to tabulate the odds ratio for the meta-analysis (On-line Table 2). The weighted averages and ranges of the extracted/tabulated sensitivities and specificities with corresponding ranges of CTP in the detection of DCI from these studies were $0.84(0.7-0.95)$ and 0.77 (0.66-0.82), respectively. The meta-analysis also revealed a pooled odds ratio of 23.14 (95\% CI, 5.87 - 91.19) (Fig 3), suggesting that the patients with aneurysmal SAH with a positive CTP test were approximately 23 times more likely to experience DCI compared with those patients with aneurysmal SAH without a positive CTP test. The study heterogeneity $\left(I^{2}=6.96\right)$ in these data was not significant $(P=.073)$.

\section{DISCUSSION}

The pathogenesis of DCI is now thought to be multifactorial, with an alteration of cerebral perfusion and reduced CBF leading to ischemia and/or infarction. ${ }^{7}$ CTP can noninvasively evaluate cerebral perfusion and can often be combined with other CT-based examinations, thereby minimizing examina- 
tion time. Few studies, however, have assessed the ability of CTP to detect DCI. ${ }^{9}$

DCI is considered a diagnosis of exclusion and is often difficult to make prospectively, especially in sedated and/or comatose patients, as physical examinations may not be reliable. ${ }^{5}$ Furthermore, defining DCI by its primary outcomes measures only allows its detection after irreversible damage has already occurred, thus limiting the timely delivery of efficacious therapy. Because DCI involves perturbations in CBF, it is thought that CTP may be able to detect DCI before its primary outcomes of cerebral infarction or functional disability manifest, thus allowing pre-emptive therapy. ${ }^{13}$

Our systematic review and meta-analysis indicates that patients with aneurysmal SAH with positive CTP test results demonstrating perfusion deficits are approximately 23-fold more likely to experience DCI compared with patients with normal CTP results, suggesting that CTP has the potential to be a useful tool in clinical practice. Compared with other imaging measures used to detect DCI, such as intraventricular hemorrhage and Fisher scores, hydrocephalus, and transcranial Doppler flow prolongation, which have reported odds ratios ranging from $0.5-$ $2.7,{ }^{26}$ CTP has demonstrated a higher odds ratio in this metaanalysis. Moreover, CTP had a superior odds ratio, even at the lower limit of the $95 \% \mathrm{CI}$, compared with other nonimagingderived parameters, such as an admission mean arterial pressure $>112 \mathrm{~mm} \mathrm{Hg}$, which has a reported odds ratio of 3.3. ${ }^{26}$ This result was achieved despite variation in the patient populations studied, CTP protocols and software used, and the various definitions of DCI implemented in the studies included in this meta-analysis.

By applying the QUADAS tool, we found that among the studies included in our systematic review, most were free of spectrum bias, primary selection bias, differential verification bias, and incorporation bias (see "Results" section for description). They each also clearly described the definition of DCI implemented and patient selection criteria used. In most cases, the choice of a reference standard was considered appropriate. Most studies, however, contained signs of disease progression bias and test classification bias (see "Results" section for description). Last, most studies did not explain patient withdrawals from the study, nor did they report uninterpretable or intermediate test results. These shortcomings represent areas of future improvement in diagnostic accuracy studies assessing the role of CTP in detecting DCI in aneurysmal SAH.

Our analysis also revealed other limitations of the existing literature on CTP for the detection of DCI. These primarily arose from the nonuniform definitions for DCI used in the literature, interinstitutional differences in the CTP protocol and postprocessing software programs used, and the lack of consistency among the definitions of an abnormal CTP test result (On-line Table 2). Given such variability, our analysis combined the various definitions of DCI used in each study. Specific subanalyses to determine if a particular definition of DCI was better detected by CTP could not be performed given the limited sample size of the studies that met eligibility criteria in this investigation. Other subanalyses examining the best time to perform CTP to detect DCI (hospital admission, during symptoms, or both) could also not be performed because of the variability of when CTP was performed among the studies that met our eligibility criteria. Furthermore, those studies that assessed CTP test results quantitatively reported test characteristics for different CTP hemodynamic parameters (MTT, CBF, CBV, and TTP), often by using various thresholds. In these cases, we chose the CTP parameter and threshold with the greatest overall diagnostic value for DCI because we were evaluating CTP as a single test and not by its individual components. These limitations, and the fact that the studies that met our eligibility criteria also had variable CTP methodology and postprocessing algorithms with evaluation of the results limited to the anterior circulation, may be sources of bias and must be considered when the results of our meta-analysis are interpreted. However, despite these differences among studies, our meta-analysis revealed a statistically significant odds ratio and no statistically detectable heterogeneity, suggesting that a strong association exists between CTP deficits and DCI, across variations in DCI definition and CTP technique used.

It is noteworthy to mention that CTP itself has limitations. The amount of brain volume coverage may be limited in the clinical setting, and the amount of radiation exposure to the patient is higher compared with standard noncontrast CT of the head. ${ }^{27}$ Furthermore, accurate quantification is dependent on an intact blood-brain barrier, which may not be normally functioning in ischemic events, such as DCI. ${ }^{27}$ Last, different postprocessing software vendors may quantify CTP data differently, which may introduce undue variation in the results. Despite these shortcomings, however, because CTP uses ubiquitous technology, it has the potential to be quickly and widely implemented. CTP, therefore, may find a place in the clinical setting for better and earlier detection of DCI and its devastating outcomes.

\section{CONCLUSIONS}

Our meta-analysis revealed a strong association between CTP perfusion deficits and the development of DCI in patients with aneurysmal SAH. Because DCI is challenging to diagnose prospectively, treatment is often of limited efficacy when initiated after morbidity has already occurred. CTP may aid in the detection of DCI earlier and with more accuracy, allowing for preemptive therapy and/or closer monitoring to prevent potentially devastating outcomes of cerebral infarction and functional disability.

Disclosures: Ajay Gupta_UNRELATED: Grants/Grants Pending: AUR GE Radiology Research and Academic Fellowship. Pina Sanelli-RELATED: Grant: This publication was made possible by Grant Number 5K23NS058387 from the National Institute of Neurological Disorders and Stroke (NINDS), a component of the National Institutes of Health (NIH). Its contents are solely the responsibility of the authors and do not necessarily represent the official view of NINDS or NIH. * *Money paid to institution.

\section{REFERENCES}

1. Graf CJ, Nibbelink DW. Cooperative study of intracranial aneurysms and subarachnoid hemorrhage. Report on a randomized treatment study. 3. Intracranial surgery. Stroke 1974;5:557-601

2. Hop JW, Rinkel GJ, Algra A, et al. Case-fatality rates and functional outcome after subarachnoid hemorrhage: a systematic review. Stroke 1997;28:660-64

3. Kassell NF, Torner JC, Haley EC Jr, et al. The International Cooperative Study on the Timing of Aneurysm Surgery. Part 1: Overall management results. J Neurosurg 1990;73:18-36 
4. Mayberg MR. Cerebral vasospasm. Neurosurg Clin $N$ Am 1998;9:615-27

5. Vergouwen MD, Vermeulen M, van Gijn J, et al. Definition of delayed cerebral ischemia after aneurysmal subarachnoid hemorrhage as an outcome event in clinical trials and observational studies: proposal of a multidisciplinary research group. Stroke 2010;41:2391-95

6. Frontera JA, Fernandez A, Schmidt JM, et al. Defining vasospasm after subarachnoid hemorrhage: what is the most clinically relevant definition? Stroke 2009;40:1963-68

7. Hansen-Schwartz J, Vajkoczy P, Macdonald RL, et al. Cerebral vasospasm: looking beyond vasoconstriction. Trends Pharmacol Sci 2007;28:252-56

8. Hoeffner EG, Case I, Jain R, et al. Cerebral perfusion CT: technique and clinical applications. Radiology 2004;231:632-44

9. Greenberg ED, Gold R, Reichman M, et al. Diagnostic accuracy of CT angiography and CT perfusion for cerebral vasospasm: a metaanalysis. AJNR Am J Neuroradiol 2010;31:1853-60

10. Liberati A, Altman DG, Tetzlaff J, et al. The PRISMA statement for reporting systematic reviews and meta-analyses of studies that evaluate health care interventions: explanation and elaboration. PLoS Med 2009;6:e1000100

11. Whiting P, Rutjes AW, Reitsma JB, et al. The development of QUADAS: a tool for the quality assessment of studies of diagnostic accuracy included in systematic reviews. BMC Med Res Methodol 2003;3:25

12. van der Schaaf I, Wermer MJ, van der Graaf Y, et al. Prognostic value of cerebral perfusion-computed tomography in the acute stage after subarachnoid hemorrhage for the development of delayed cerebral ischemia. Stroke 2006;37:409-13

13. Dankbaar JW, de Rooij NK, Rijsdijk M, et al. Diagnostic threshold values of cerebral perfusion measured with computed tomography for delayed cerebral ischemia after aneurysmal subarachnoid hemorrhage. Stroke 2010;41:1927-32

14. Sanelli PC, Ugorec I, Johnson CE, et al. Using quantitative CT perfusion for evaluation of delayed cerebral ischemia following aneurysmal subarachnoid hemorrhage. AJNR Am J Neuroradiol 2011; 32:2047-53

15. Dankbaar JW, de Rooij NK, Velthuis BK, et al. Diagnosing delayed cerebral ischemia with different CT modalities in patients with subarachnoid hemorrhage with clinical deterioration. Stroke 2009;40: 3493-98

16. Sanelli PC, Anumula N, Johnson CE, et al. Evaluating CT perfusion using outcome measures of delayed cerebral ischemia in aneurysmal subarachnoid hemorrhage. AJNR Am J Neuroradiol 2013;34: 292-98
17. van der Schaaf I, Wermer MJ, van der Graaf Y, et al. CT after subarachnoid hemorrhage: relation of cerebral perfusion to delayed cerebral ischemia. Neurology 2006;66:1533-38

18. Dankbaar JW, de Rooij NK, Smit EJ, et al. Changes in cerebral perfusion around the time of delayed cerebral ischemia in subarachnoid hemorrhage patients. Cerebrovasc Dis 2011;32:133-40

19. Rijsdijk M, van der Schaaf IC, Velthuis BK, et al. Global and focal cerebral perfusion after aneurysmal subarachnoid hemorrhage in relation with delayed cerebral ischemia. Neuroradiology 2008;50: $813-20$

20. Sanelli PC, Jou A, Gold R, et al. Using CT perfusion during the early baseline period in aneurysmal subarachnoid hemorrhage to assess for development of vasospasm. Neuroradiology 2011;53:425-34

21. Killeen RP, Mushlin AI, Johnson CE, et al. Comparison of CT perfusion and digital subtraction angiography in the evaluation of delayed cerebral ischemia. Acad Radiol 2011;18:1094-100

22. Lagares A, Cicuendez M, Ramos A, et al. Acute perfusion changes after spontaneous SAH: a perfusion CT study. Acta Neurochir (Wien) 2012;154:405-11; discussion 411-12

23. Harrigan MR, Magnano CR, Guterman LR, et al. Computed tomographic perfusion in the management of aneurysmal subarachnoid hemorrhage: new application of an existent technique. Neurosurgery 2005;56:304-17

24. Sviri GE, Britz GW, Lewis DH, et al. Dynamic perfusion computed tomography in the diagnosis of cerebral vasospasm. Neurosurgery 2006;59:319-25

25. Pham M, Johnson A, Bartsch AJ, et al. CT perfusion predicts secondary cerebral infarction after aneurysmal subarachnoid hemorrhage. Neurology 2007;69:762-65

26. Claassen J, Bernardini GL, Kreiter K, et al. Effect of cisternal and ventricular blood on risk of delayed cerebral ischemia after subarachnoid hemorrhage: the Fisher scale revisited. Stroke 2001;32: 2012-20

27. Latchaw RE, Yonas H, Hunter GJ, et al. Guidelines and recommendations for perfusion imaging in cerebral ischemia: A scientific statement for healthcare professionals by the writing group on perfusion imaging, from the Council on Cardiovascular Radiology of the American Heart Association. Stroke 2003;34:1084-104

28. Reichman MB, Greenberg ED, Gold RL, et al. Developing patientcentered outcome measures for evaluating vasospasm in aneurysmal subarachnoid hemorrhage. Acad Radiol 2009;16:541-45

29. Reichman M, Gold R, Greenberg E, et al. Validation of a new reference standard for the diagnosis of vasospasm. Acad Radiol 2010; $17: 1083-89$ 